\title{
Language, Culture and the Neurobiology of Pain: a Theoretical Exploration
}

\author{
HORACIO FABREGA, JR. \\ University of Pittsburgh, School of Medicine, Department of Psychiatry, Pittsburgh, Pennsylvania \\ 15213, USA
}

\begin{abstract}
Language and culture, as conceptualized in traditional anthropology, may have an important influence on pain and brain-behavior relations. The paradigm case for the influence of language and culture on perception and cognition is stipulated in the Sapir-Whorf hypothesis which has been applied to phenomena "external" to the individual. In this paper, the paradigm is applied to information the person retrieves from "inside" his body; namely, "noxious" stimuli which get registered in consciousness as pain.
\end{abstract}

\section{Introduction}

Every person seems to "know" what pain is and by means of language is able to describe it. Given the ubiquity and importance of pain in the adaptation of higher animal forms, one may infer that it has played an important role in evolution. It is thus very likely that pre-hominids and earlier members of the human species also "knew" a great deal about pain. A neurophysiologist would claim that pain is based on brain structures which all members of the human species share. At present these structures and their mode of functioning are incompletely understood. An anthropologist who endorses a position of cultural relativism is aware of the variety of beliefs and understandings about pain and behaviors associated with it and would claim that there appear to exist not one but many varieties of pain (Fabrega, 1974; Zborowski, 1958; Fabrega and Tyma, 1976a,b).

Language and culture play some role in the phenomenon of pain. Their influence raises interesting scientific and philosophical questions about human adaptation which need to be heeded. As an example, experimental pain needs to be studied under highly controlled conditions using animals. When human subjects are used in studies on the physiology of pain, then they usually come from urban and complex nations. When researcher and subject are from the same background, the possible role of culture and language is circumvented. A source of potential variability in behaviors surrounding pain is thus not amenable to study. Similarly, the exigencies of having to treat clinical pain very often force the physician to concentrate on factors which he can modify. This has the effect of reducing pain to wellestablished principles of physiology and psychology (e.g. anxiety, personality). In either case, language and culture as traditionally conceptualized by $0953-4180 / 89 / 040235+26 \$ 3.50 / 0 \quad$ (C) 1989 CNS (Clinical Neuroscience) Publishers 
anthropologists tend to be under-emphasized in analyses of pain. How do the perspectives which the neurobiologist and cultural anthropologist have about pain differ and in what ways are they related? In the present paper this and related questions are explored.

\section{Background Considerations: The Sapir-Whorf Hypothesis}

Anthropological studies of people from different cultures who speak different languages have raised the question whether these differences make cognition and perception different. This question lies behind the SapirWhorf hypothesis which suggests that different representations of the world may exist and along with this different ways of thinking about it and different styles of problem solving. Possible differences in perception and cognition are believed to stem from differences in the system of language and associated cultural factors. The hypothesis was propounded following studies with the Hopi people (Whorf, 1964; Sapir, 1927) who do not overtly mark tense (a feature of language) as occurs in Indo-European languages. Persons who spoke Hopi were said to be unable to "objectify" time. Anthropologists speculated that a way of describing time through language influenced how the Hopi perceived and experienced time and, more generally, how time entered into their planning and reasoning (a feature of thought). Inferences about cognition, however, were initially drawn purely from the study of language itself. This circularity led researchers to emphasize that non-linguistic evidence of cognition was required for verifying the hypothesis that language might affect thought. Fishman (1960) surveyed the history of the Sapir-Whorf hypothesis and provided a useful systematization of its features. He drew attention to the separate and distinct components of the hypothesis, each of which needs to be measured independently. These involve features of language (e.g. lexical, semantic, or grammatical) and features of thought as reflected either in language (cultural themes, myths) or non-linguistically (e.g. memory).

The Sapir-Whorf hypothesis can be formulated in two ways. The first is: Are language habits and rules, which may differ across cultural groups, associated with how people actually perceive the world and how they actually think? The second question is: Do the language habits and rules of a people actually cause and determine different ways of perceiving and thinking? The first question touches on the linguistic relativity hypothesis, the second on that of linguistic determinism. Evidence for linguistic determinism awaits longitudinal developmental studies. On the other hand, although there exists evidence suggesting linguistic relativity, its exact interpretation is contested (Cole and Scribner, 1974; Heider, 1972). The relation between language and thought has not yet been clarified.

The possible influence of language on thought is formulated in a generic manner. In other words, any feature of a language can be related to any feature of cognition, and a relation between the two might exist in any domain of experience to which language and cognition are applied. In early 
investigations, the strategy appeared to be to first make explicit, through linguistic analyses, properties of a particular language (e.g. its lexical items, grammatical rules). A language which differed in the way a similar property was realized was then chosen for comparison. Thus, languages which seemed to (lexically) name things differently or whose grammars required users to focus on different components of events and processes (e.g. duration, shape, relation to self) might be chosen. Once contrasting languages were selected, a cognitive task was devised which, it was assumed, would allow the chosen property of the language to differentially assert itself (e.g. in memory, problem solving, etc.). This would mean, as an example, that groups of speakers of each language would be compared in terms of how they classified entities or how well they coded and remembered them, with the expectation that one of them would perform better because their language would "facilitate" the task. It was thus necessary to compare groups on tasks involving a distinctive aspect of experience.

In early evaluations of the Sapir-Whorf hypothesis, the color domain proved a popular one. Since several forms of physical continual (e.g. hue brightness, saturation) could be used to independently code the color spectrum, this allowed precise determination of the location and boundaries of native color terms. One could thus determine which physical colors (e.g. instanced in color chips) were being "mapped" or named by the native color terms. Investigations of the role of language on the perception of color seemed to be undertaken with the following assumption: The color spectrum is arbitrarily partitioned by the native color terms of a language system (i.e. it is "cut up" purely by convention). In other words, any color area may be singled out or "mapped" by a color term, and in different languages and cultures altogether different (and arbitrary) color areas would be named by different color terms.

The history of the problem of the perception of color has been recently reviewed (Berlin and Kay, 1969; Bornstein, 1973, 1975; Sahlins, 1976). Opinion has shifted from a position which ascribed to culture-language factors (e.g. types of native color words) an important and influential role in the process of perception (linguistic relativity), to one which ascribes to perception an influential role on culture and language (the perspective is termed phenomenal absolutism). The latter position is that species-wide neuro-anatomic structures and physio-chemical processes underlie the sensation and perception of color and that such neurological substrates have a determining influence on how an individual responds to color, codes it through language, and retrieves it in its memory system. In brief, built in "organic factors" subserving central visual processes are influential in how color is understood, what it means, and what color terms signify in the phenomenal world. Related but not identical uniformities in neurological structures and functions characterize infra-human species so that one can say that among them also organic factors serve to control how color is perceived and thus how it can influence behavior. Culture-linguistic differences involving categorization of hues and color nomenclatures (specifically in the yellow and blue region) have been described among Negroids 
and Mongoloids and these are believed to stem from differences in retinal pigmentation which themselves are related to geographic setting. In this instance, biological diversity of peripheral visual processes underlie the observed language-culture differences.

Sahlins has argued that biological uniformities subserving the neural processing of color in no way undermine the distinctiveness of color viewed culturally (Sahlins, 1976). Such uniformities merely create the boundaries and distinctions which language and culture require in order to render color meaningful to a people. The culture-language (in his terms, the symbolic or semeiotic) significance of color lies not in the similarities of recognition of basic natural colors but in the relating of these through color terms to each other and to the myriad of symbols about people, things, events, processes, etc. which a people regard as significant. In brief, the physical correlates of basic color words comprise a set of distinctive features out of which differences in meaning are built. In his argument, Sahlins appears to be saying that the Sapir-Whorf hypothesis does not apply to neural processing and/or that verification of the hypothesis lies not in documenting differences in the way people point to color but differences in the way color is used by them to regulate social behavior.

The analysis in this paper explores the possible influence of culture and language on an equally important area of human functioning, namely pain. For comparative purposes, the physical spectrum of wave lengths underlying the perception of color can be analogized to varieties and amounts of physical stimulation which impinge on sensory receptors and generate activity in the nervous system which can give rise to pain. A people's color terms can be equated with their pain terms (and the way these are used) when they describe pain and social psychological behaviors related to linguistic/cultural understandings of color to social psychological behaviors subserving pain. Instead of how man codes, represents, and organizes the external world by means of the dimensions of hue brightness and saturation one is concerned with how he codes, represents and organizes phenomena in his internal world which involve varieties and intensities of noxious stimulation. There is a large difference between color and pain. A color chip is a physical token which one equates with a color term which in turn is used to create diverse overtones of meaning about significant things in the world. However, a specific variety and quantity of nociceptive stimulation is but a physical analogue of a special type of stimulation which is perceived as an altered condition of the self. In short, through color one articulates cultural meanings whereas in pain one experiences oneself as meaningfully altered. This difference makes the study of pain from a culture-language standpoint interesting and also gives rise to special considerations.

\section{Culture, Language and Pain: Behavioral Considerations*}

The members of a culture group orient and behave consistently with their views about the world and these views are mirrored even in the structures *I am grateful to Steve Tyma for help in clarifying some linguistic aspects. 
and systems of their languages (Pike, 1967). Compared to other domains of experience pain is different since it is internal, hidden and private. Shared conceptions of pain are forged through the medium of language. Experiences of pain come to be described and interpreted by means of this language. An individual learns the lexical and phrasal units that are appropriate to events he often cannot observe, and both linguistic and nonlinguistic behavior constitute the means of communicating about and ultimately confirming his perceptions. As a consequence, the selective and interpretive qualities of a people's culture and language are particularly salient in its pain behaviors. Because of these considerations one is able to claim that pain viewed in a cultural context is shaped by language and related symbolic factors.

The influence of language abstracted from its cultural context can be traced to the formal properties (the "structure") of a linguistic event such as a monologue, a dialogue, a discourse or some other utterance. In the present context, the linguistic events subjected to scrutiny would be pain descriptions and all of their relevant lexical, syntactic and semantic properties. For example, the Thai language employs several basic or primary pain terms, the morphological differences of which reflect the presumed origins and locations of pain experiences. Additionally, Thai has a pain term bit ("twisted") which metaphorically qualifies pain experiences (e.g. as in "menstrual cramps"). Presumably, a Thai native healer accommodates his treatment to the description of the phenomenon to be treated. Japanese has but one basic root form (morpheme) to describe pain, and realizations of "pain" (ita-) in reports of pain employ derivational affixes. In Japanese, syntactic distinctions which are realized by derivational affixes are extremely important in pain descriptions. English has four basic or primary pain terms (pain, ache, sore, hurt) which are portmanteau realizations of semantic information (Pain) and syntactic information (e.g. in American English, sore is either an adjective or a noun, but never a verb). Each of the four primary pain terms of English can connote intensity and associated emotional factors but they do not state the source nor position of the pain. The history of English pain terms, as well as it can be documented, provides clues about how the phenomenon of pain has come to be conceptualized. Through linguistic analysis these observations suggest differences in cultural constructions of pain; behavioral differences are implied but pain behavior has not been subjected to rigid structural analysis (Fabrega and Tyma, 1976a,b).

Descriptions of pain experiences are enriched by other, "secondary" terms. For example, English relies on change-of-state verbs (e.g. crushing, cutting, burning, jumping) for metaphoric qualification in pain descriptions. Other languages could rely on the same type of pain metaphors, but only insofar as such is seen as a "natural" circumstance. A culture employs only those linguistic devices (e.g. syntactic inflections, semantic roots) that are deemed consonant with its conceptualizations of the phenomena for which the devices are employed in descriptions. The underlying conceptual models of pain which are realized in verbal descriptions differ sharply.

Language communities may avail themselves of a number of linguistic 
devices such as nominalization, metaphor, simile, etc., but the use of these devices is subject to any of a number of language-specific constraints. For example, a Thai speaker does not normally speak of his pain as an entity or thing; Thai pain terms lack the nominal forms that are available in English or Japanese. An English speaker may claim that he "has" ("owns" or "possesses") a pain condition or quality, and through his selection of a primary pain term, he can convey different facets of his experience (e.g. intense pain versus mild discomfort, recent injury versus an old condition, etc.) A Japanese speaker may make similar reports of the facets of his pain experiences, but he does so through qualifications grounded on the single primary pain term available to him. An English speaker can report having a "knot" in his stomach or a "cramp" in his leg because twisting, turning and cramping are acceptable metaphors shaped by a conceptualization of pain that further validates a view of pain as a "thing" (nominalization) and a physical deformation (change-of-state). Different metaphors are available in each language and the acceptability of metaphors varies since analogies are in part attributable to properties selected by language and culture patterns. A language community may have to rely on simile (e.g. "It feels like I have a knife inside"), but again the appropriateness of comparisons made through simile may in some sense be attributable to conceptualizations of pain. Japanese employs a sound symbolism in describing pain, but this sound symbolism is identical to that found in other patterns of the Japanese language (as in those words used to denote regularity or frequency). In Japanese, the subjective and emotional aspects of pain seem to be all important and this is communicated through the images aroused by phonetic patterns. Thai does not seem to employ metaphor or sound symbolism for secondary qualification in pain descriptions; one can speculate in the face of this kind of evidence that purely psychological aspects of pain are less critical to verbal descriptions of pain in Thai.

From the way people use their pain terms one can infer that specific types of "grammatical" constraints are operating. These constraints differ in the patterning of such elements as time, causality, substance, change, conceptualizations of self, and forms of possession or ownership. The premises in terms of which the phenomenon of pain is understood are implicitly contained in the pattern or system of descriptions of pain. The denotation of a culture's premises of pain is embodied in those terms which are central to pain descriptions, for it is these terms and their syntactic configuration (usage and constraints) that indicate the conceptual dimensions of pain. Some terms are primary to pain descriptions in that utterances in which primary pain terms do not appear cannot be said to describe "pain" exclusively. The connotation of a culture's premises of pain is embodied in those terms which qualify or limit pain in descriptions. The primary pain terms illustrate a semantic range for the main dimensions of a pain experience and the secondary pain terms further narrow the qualities that the pain experience is seen to have. But, secondary pain terms do not refer exclusively to pain. Because they differ formally and syntactically across languages, pain descriptions can be seen as incorporating different 
concepts of causality, change, and concreteness as well as the relationships between the self and the experience of pain or its presumed source.

The organization and meaning of pain descriptions can be equated with the organization and meaning of the behavior of an individual who is in pain. Pain descriptions may be assumed to reflect and in a sense control the premises through which a culture is oriented to the world and their organization and meaning underlies and/or is mirrored in human social behavior. It should be possible to analyze non-verbal behavior as communication and synthesize the formal, "grammatical" (structural) features of such nonverbal behavior as gestures, facial expressions, and other activities. The linguistic and nonverbal symbols through which all facets of life including pain are rendered meaningful by a people make explicit views about the nature of the person, physical and animate matter, worldly and other worldly (cosmologic) governance and rules of social conduct.

Language and symbolic systems generally are bound together by internal forces of cohesion that regulate notions of contrast. (Pike, 1968, 1977). This cohesion is necessarily internal because symbols are meaningful only in a context. Conclusions drawn from studies directed at the discovery of universal pancultural features of any purposeful behavior are useful for any number of reasons; as an example, for understanding communication as a process. However, such studies as a matter of course must minimize the degree to which a distinctive cultural tradition relies on its own specific system of paradigm and syntax consonant with premises about the nature of the world in which the members of that culture must live. It is therefore, possible to observe, as an example, that the English body part names heart and arm contain nuclei that are phonetically similar. This phonetic similarity confirms a metaphoric relation between heart and arm. The heart is an organ the function of which is critical to maintenance of the life support of the human body. In Anglo-American culture, the arm is seen as critical to the performance of human tasks and also tasks which are deemed crucial to the maintenance of certain elements of human society; this sense of performance is reflected in the words farm and army, where the role of the formative arm is central. It can be said that the shapes of the English words heart and arm suggest that in some senses they are similar, and this similarity is borne out of the relevance of these terms to a field of reference. On the other hand, other features of these words suggest a contrast between heart and arm that indicates that the two body parts are distinct. Some of the features that distinguish the word heart from the word arm (i.e. the initial and final consonants) can be found in the initial consonant and the vowel of the word head. The occurrence of these features in both head and heart implies a degree to which these two body parts are seen as similar. The relation between these and other Anglo-American body part names suggest a folk theory about the interrelatedness of some features of the body as they are seen from a particular cultural perspective. This type of similarity of form and meaning can likewise be found in the interrelation of the primary or secondary pain terms in some languages. A language's pain terms may contain recurrent phonological elements, or be very clearly related to other phenomena. In 
Anglo-American culture, there is a very clear connection between the conceptualization of pain and punishment (Fabrega and Tyma, 1976a,b).

The formal-semantic properties of the descriptions of pain reflect presumptions about the source or raison d'être of the pain. A pain description implies a model of the cause and significance of the pain (e.g. physical trauma, failure to observe a taboo). In these implied symbolic events surrounding pain, beliefs about what takes place in the body must be assumed to play some role in affecting the quality and perhaps intensity of pain and in governing the behavior of people who report it (Wolff and Langley, 1968; Fabrega and Manning, 1973; Zola, 1966; Lewis, 1975). Other forms of behavior such as gestures and facial expressions associated with a particular mode of pain description are likely to be influenced if not structured in a manner consistent with the construal of the experience. The relationship between pain descriptions and pain behavior are analogous to the correlations between descriptions and behaviors involving syndromes termed "hysterical" or "psychogenic" (Ruesch and Bateson, 1968).

The symbolic code through which conceptions of the body are expressed differs from culture to culture, and partly in accordance with the degree of access that members of the culture have to the body. Such access is gained, for example, through various ritual procedures such as slaughter of game, autopsy or funeral customs. The number and variety of words which a language has about the body reflects the extent to which the body is subjected to analysis (Franklin, 1963). Some cultures, for example, do not recognize a difference between some body parts recognized in other cultures, and such a distinction may also be found in the terms used to name the parts or substances of the body. Where English has two words arm and elbow which indicate a distinction between the two body parts, some languages may use the same word for both the arm and the elbow to indicate that the two are not considered different. How and to what extent pain behaviors associated with disturbances in these anatomical parts would be affected is not known. As noted for the English words heart and arm, some lexical similarities underscore semantic consistency, but other phonetic features indicate that even though some terms are complementary or somehow similar, they are none the less different in important respects.

Cultural groups also differ in explanations of body functions and of how the body can become diseased or disordered (Fabrega, 1975). Such differences give rise to additional facets of pain behavior. The breadth of a cultural belief system conceptualizing the abdominal viscera (or any anatomical region) may be reflected in the behavior of people of that culture when their viscera are stimulated or diseased. Similarly, pain behavior can reflect views about causal agents and processes believed to change viscera during disease. Features of pain behavior restricted to a particular cultural group may be related to whether the agencies of disease are viewed in association with personal actions of self and/or co-members (i.e. caused by disrespect to gods, witches, etc.) or impersonal, mechanical influences (i.e. having no intrinsic social rationale). It is certainly likely that conceptualizations and with this, pain behaviors of different sorts, are altered as theories of pain, disease and bodily function become more technical, impersonal 
and more differentiated. The influence of language and culture on conceptualizations of pain, and associated behaviors, has an obvious relation to conceptualizations of bodily experience and to the phenomena of somatization.

\section{Cultural-Linguistic Differences and Brain-Behavior Relations}

People of different groups behave differently, speak different languages, and understand the world differently. Yet, as members of one species they inherited one type of brain. Are behavior differences correlated with neurobiologic differences? How might the environmental influences associated with "culture" affect brain function?

In attempting to understand the influences of culture and language on brain-behavior relations at least four sets of factors must be identified. The first one involves the nature of the stimulus. It is trivial to say that members of different cultural groups will respond differently, neurologically or behaviorally, to physical stimuli or patterns thereof. A critical question is which stimuli and how one goes about testing these. Members of different nations will respond differently to physically identical flags, just as members of simple societies will respond differently to physically identical animals (whose meanings differ). Here the differences include, besides "physical stimuli", obvious symbolic factors involving cognition, values and emotion. The physical stimuli provided by a flag or ritual object produce far more than "sensory registration". The neural consequences of such stimuli will involve cortical and subcortical structures which are necessary for the realization of meaning and emotion. In light of the difficulty of measuring complex and widespread neural responses such as these, one interested in specifying possible culture-linguistic effects on brain-behavior relations is compelled to better control and standardize the stimulus itself. Ultimately, standardization is best achieved with a discrete type, intensity and duration of physical energy. However, much of what social scientists find interesting and important involves responses to "complex" patterns of stimuli; precisely such things as flags, ritual objects or states of disease producing pain. The type of stimulus most useful for clarifying differences in brain-behavior relations therefore presents obvious problems.

A second set of factors involves the nature of the behavior or response which will be taken to reflect neural events. How broadly one chooses to define "pain behavior" and what indicators will serve to measure it will differ depending on the orientation of the scientist. A researcher who is holistic is likely to insist on a large and "meaningful" segment of behavior to analyze. A physiologist is likely to first search for discrete behavioral responses which can be measured precisely and which may be taken to unambiguously reflect nervous system functioning. In the case of noxious stimulation, verbal statements might be sought regarding discrimination or intensity (pain threshold, pain tolerance) since these are "closest" to the changes taking place in the neural structures which are believed to subserve pain. In using verbalizations or judgements as indicators of nervous system functioning, the physiologist is likely to resort to certain "psychophysical 
linking hypotheses". An example of this, was provided by Brindley (1960): "... whenever two stimuli cause physically indistinguishable signals to be sent from the sense organs to the brain, the sensations produced by these stimuli, as reported by the subject in words, symbols, or actions, must also be indistinguishable". By means of this hypothesis the sensory physiologist will allow that if a subject (or animal) cannot distinguish between two stimuli, it is reasonable to hold that these stimuli are producing similar sensations (i.e. neural events). This psychophysical linking hypothesis, it should be noted, applies for sensations taking place in one organism (i.e. in the "same" nervous system). This type of hypothesis may no doubt be modified so as to admit observations and experiments conducted on two different nervous systems (i.e. in persons from two cultural groups). In this instance, then, the response unit or segment which is to be taken as a possible neural correlate of culture or language is simple: a judgement of indistinguishability (or a behavior which reflects this) vis à vis a highly controlled stimulus. To a cultural anthropologist, such a unit of behavior is not critical; it is how pain is appraised and what effects it has on social behavior which would be deemed important. On the other hand, the prospect of studying the neural correlates of the concept of pain and the meanings of pain behavior in a symbolic or semeiotic framework would baffle a neurophysiologist.

A third set of factors involves the "unit" (or level) of the nervous system which could be held to possibly reflect the influences of culture linguistic variables. Differences in pain behaviors which span social and cognitive spheres can involve sensory processes (registration of stimuli), intracortical connections (so called intermodal associations), language structures (verbal mediation) and limbic system activation (reward, drive, motivation, etc.). Establishing group differences in pain behavior simply indicates that the nervous system as a whole is activated differently. Whether the differences reflect structural or physiological changes in discrete neurons, intercellular connections, or larger cell assemblies is obviously unspecified. Yet, neural changes like these are the ones neurophysiologists would want to demonstrate in order to make a strong claim about the effect of language-culture on the way the brain functions. The basic task is to show that as a result of a particular type of cultural upbringing, a brain structure functions differently. To accomplish this, one must identify possible structures which are to be studied, specify in a precise and rigorous way what the neural inputs are, and then show that function in them bears the effect of language and culture; specifically that people from different cultural groups encode or process neural information differently. It is for this reason that responses in well specified neuro-anatomical structures are chosen for study (e.g. evoked responses in the occipital or somato sensory cortex). Again, responses desired by the neurobiologist are divested of that which is of interest to the social and cultural anthropologist since to the former neural responses only have meaning in the Western scientific framework and not in a semeiotic one. It is not clear whether a cultural anthropologist would claim that neural responses are totally inappropriate or whether he would demand that 
the process of compartmentation of structure and function not be a reductionist.

The final set of factors concerns the fixity and permanence of any nervous system property due to language-culture. No one doubts that individuals, regardless of their culture, can be taught different skills or habits. Insofar as behavior is a product of (or regulated by) the nervous system, it follows that such learning is somehow coded in the nervous system. In this light, one can ask: Are the modalities of nervous system organization and function which lie behind "cultural" learning enduring? Can they be modified? Can persons not of that culture learn equally well the respective behaviors and can it be shown that similar modalities of neural functioning prevail? In the study of culture-language behaviors and their neural analogues, then, the modifiability and stability of the way a neural structure is organized and functions is important. To show general differences in easily modified (and/or approximated) behavior routines and associated neural happenings across cultural groups is to demonstrate again that people can learn equivalent tasks. To show enduring and/or permanent forms of neural representation and functioning in distinctive structures or brain areas across cultural groups is (if these are culturally acquired) to show that culture and nervous system functioning have interacted in an elemental way.

The preceding four sets of factors involved in the study of languageculture influences in nervous system functioning - the nature of the stimulus, the behavioral response, the neural response, and the modifiability of the neural mechanism - bear a relation to each other. By scientifically comparing (in people from different language-cultural groups) ever more precisely controlled stimuli, reliably recording discrete items of behavior the meanings of which are clearly spelled out, linking these to neural changes in specific units whose inputs are known and controlled, and demonstrating that the neural changes are both distinctive and stable, one shows that language-culture has affected in a fundamental way the structure and function of the nervous system. In the preceding sentence, the term "language-culture" can be substituted by "experience" or "an environmental factor". The result becomes a sentence of general relevance in the field of neurobiology. The special problems posed by the study of language and cultural influences on the neurobiology of pain can now be considered.

\section{Culture and Language in a Neurobiologic Model of Pain}

Difficulties are encountered when one attempts to relate culture and language influences to brain mechanisms which mediate pain. First of all there is the philosophical problem of mind/body relations. A central element of any discussion of "pain" concerns subjective experiences which by definition are private, "mental" and removed from spatio-temporal analyses. But the analyses of the neurobiologist concern spatio-temporal factors, 
e.g. concrete structures, pathways, electrical (neural) impulses and chemical reactions. To avoid the trap of the mind/body dilemma, such things as the "experience" and "perception" of pain should be minimized in favor of observable aspects of behavior (verbal and nonverbal).

For purposes of discussion, one can construct a model of pain which includes a (1) peripheral nerve and sensory receptor component, a (2) spinal cord component (e.g. class 1 and 2 dorsal horn sensory interneurons), a (3) brain-stem diencephalon component (e.g. an area in the reticular formation concerned with neural registration of pain, a midbrain central grey matter area involved in analgesia, etc.) and (4) a forebrain component (e.g. somatosensory area, language area, limbic-septal areas, etc.) (Mayer and Price, 1976). As impulses conveying nociceptive stimulation travel up the neuraxis, divergence occurs and complex connections between centers having feedback loops come into play making it ever more difficult to "isolate" neural substrates subserving pain. The bulk of research on the neurophysiology of pain has concerned events taking place between the peripheral nerve and brain-stem. Conditions of pain inhibition, in both animals and man, have been related to these levels of the neuraxis. Comparatively less is known about the role and function of cerebral cortex in human pain behavior (Liebeskind and Paul, 1977; Nathan, 1973).

Consider how pain has been viewed in this neurobiological mode. An orienting assumption seems to be that pain is an outcome of the balance of neural activity in the nervous system. Thus, afferent interactions and descending controls among the systems 1-4 are somehow responsible for pain (i.e. facilitation and inhibition among systems). In this model, pain inhibition is said to occur through modulation of any of the systems 1-4.

Consider now how language and cultural factors might be related to this neurobiologic model of pain. Intuitively, one is first led to see language and cultural influences as forms of psychological influence, akin to suggestion, trance and instructional set (Hilgard, 1973). The neurological mechanism underlying these conditions of pain and pain inhibition are not at all clear. It is not known, for example, whether the inhibition takes place mainly at cortical-subcortical levels or whether some inhibition also involves transmission at spinal cord levels. None the less, it is obvious that centers mediating language and understanding (e.g. temporal lobe) are involved, and that these probably influence the brain-stem. Insofar as trance, suggestion, etc. involve communications and/or a meaningful context, they properly can be said to implicate "language and culture". However, "language and culture" is here being handled simply as a term which refers to learning, understanding and/or suggestibility: a cortically mediated capacity common to the species and seemingly devoid of symbolic specificity. It is true that research has indicated that distinctive components of language such as semantic categories may have a separate neuropsychological status and given that these may have a conventional basis (i.e. that culture-language differences exist), questions about brain/behavior differences can be raised (Warrington, 1975). Similarly, insofar as much of "cultural" behavior involves the use of "distinctive" knowledge, information somehow "in a person", the 
neurology of memory is necessarily implicated in any view of culture and language influences on pain. If an anthropologist-linguist should consent to view pain neurologically and to search for culture-language effects in the brain, he most likely (as a holist) would claim that pain involves the operation of the nervous system as a whole. A challenge from a locationist (e.g. a report about stimulation produced analgesia) would be met with the claim that electrical activation (or inhibition) of a brain structure which modifies pain level in no way invalidates the view that pain behaviors, which owe their rationale to cultural factors, are a product of an integrated brain. These and related issues about brain-behavior aspects of culture have been discussed elsewhere (Fabrega, 1977; 1981).

Several points follow from this discussion. The first is that language and cultural influences as discussed in this paper can be viewed as more or less "permanent" and as essentially "programmed" in an individual. In this sense they differ from the usual psychological influences studied by behavioral scientists (influences easily manipulated by the experimenter) which are by definition transient and reversible (i.e. "mental sets"). For this reason, studying language and culture influences in the neurobiology of pain is analogous to the study of the influences of other psychological traits which undoubtedly have neurological substrates though these may be poorly understood (e.g. personality). Construing language and cultural influences in this latter way merely points to a fundamental dilemma for the researcher which stems from his apparent quest for the neurological substrates of culture and how these function in the brain. To give credence to this quest one must first construe "culture" as "culturally distinctive behavior" and then isolate from this general category elements of behavior linked to culture (e.g. language, knowledge of rules, patterns of socially appropriate behavior, etc.) which one might possibly relate to a brain structure or modality of function. When this is tried, one finds that it is difficult indeed to point to behaviors which do not bear the imprint of culture! Categories of behavior which one may devise in an attempt to study "culturally specific behaviors" are likely not to be mutually exclusive and furthermore, insofar as these are made to embrace human action in a social context it is unlikely that any of them can be linked in a one-to-one fashion to distinct brain area or centers given the "connected" character of neural activity. In attempting to explain language and cultural influences on the neurobiology of pain one is thus forced to merely suggest general ways in which such influences might be realized.

Recall that the culture and language of a people can reflect different interpretations about the body, with different parts being accorded more importance in the definition of self. In referring to the body, then, subjects might be required to habitually treat certain parts and stimulations differently, depending on their proximity to that which was judged as the central essence of the person. Noxious stimuli arising in different body parts might consequently be interpreted and/or tolerated differently. As an example, in English "heart" (derived from the Latin cor) means far more than an organ for it conveys the notion of centeredness (e.g. the heart of the matter, the core 
of the problem). This literally means that a pain in the chest (where the heart is believed to be situated) conveys physical harm (given our model of pain) to the "core" of an individual. People of language communities which culturally and linguistically ascribe less significance to such an anatomical part may very well behave differently in the event of noxious stimulation in it. This suggests that myocardial infarctions may display different behavioral paths among people of different language communities and also that angina may provoke different types of pain behavior.

The basic point is that the heart, as an anatomical structure, is no doubt a universal "entity". As a cultural "structure", however, the heart cannot be judged a universal. In a symbolic sense, people view such an anatomical part and its region differently; it enters into their construction of themselves differently and its properties can be related metaphorically to different domains of experience. Moreover, the heart as a linguistic structure is differently constituted; this is to say that the actual word for the anatomical part will differ across language communities as will the linguistic units which make it up. The role which such linguistic units play in the lexicon (like cor in heart, core, courage, encourage, discourage) is dictated by grammatical rules, and these rules reflect a distinctive construction of the world which itself is consistent with the meanings which link the portion of the lexicon bound by the units. It is a structure such as that inherent in our view of "heart" which linguists partly have in mind when they speak of the unconscious patterning which culture and language have on experience. Such a patterning between language and culture can be held to shape the way one learns to respond to bodily stimulations and this suggests that stimulations arising in different bodily parts will be subject to different sets of culture linguistic influences.

Since the "meaning" of body parts or regions can differ, then the significance of stimulation in such parts also might vary. A consequence of this might then be that an altogether different profile of psychophysiologic measures could be activated when such body parts are stimulated (Mandler, 1967; Beecher, 1959). In an effort to study the possible influence of language and culture on the neurobiology of pain, the following kinds of questions become relevant. Do noxious stimuli arising from a particular bodily region produce unique behavioral responses regardless of language and cultural background? Do pathological conditions in various bodily organs compromise problem solving, motor organization and other components of adaptive behavior similarly, regardless of cultural and language background? Do individuals of a particular cultural or linguistic group, when compared to another, produce the same hierarchy of physiologic responses given equivalent physical stimulation in an organ system? In order to answer questions such as these it would seem that one must conduct studies among people whose culture and language provide different interpretations of "body".

Experimental studies of pain usually involve bringing an individual into a laboratory. Technical equipment is employed and suitably attired researchers or their technicians approach the "subject" in a controlled and stereotyped manner. Exposure to the laboratory is stressful and indeed is usually "controlled". Pain is assumed to have a "sensory threshold" and 
persons are asked to report on this even though they may not, strictly speaking, consider the sensation "painful". Persons are also assumed to have a level of tolerance which they themselves are allowed to set. Moreover, the whole context of the experiment suggests that any pain which may be elicited is a graded condition which can be monitored and regulated at will. The effect is to render the experience artificial and mechanical. Moreover, to the subject there is compelling evidence that any pain which is produced is caused by the instruments, which also has the effect of shaping pain along the lines of the experimenter's model. The stipulations implicit in this whole approach are of course logically required in order to study phenomena scientifically and rigorously, and criticisms about the relevance of experimental procedures in the study of pain miss the mark (Beecher, 1959). Yet, someone intending to make inferences about the role of language and culture in brain-behavior relations would insist that the neurophysiologic model of pain not totally shape the data. Stimulations capable of causing pain should be administered but under conditions which more nearly approximate natural circumstances. Finally, rather than requiring that subjects respond in terms of the researchers' model of pain, more unstructured and freely elaborated pain behaviors and descriptions should be allowed. In this way, a more accurate picture of the way culture and language influence behavior might be obtained. It is doubtful whether the neurobiology of pain in relation to cultural and linguistic factors could ever be studied in a way that allows for the free and unstructured expression of (a culturally valid) pain.

\section{Language and Cultural Influences: Ontogenetic Factors}

The preceding discussion has pointed to ways in which one may view the effects which language and culture have on the neurobiology of pain. This raises the question of how such effects may come about.

Cultural rules for behavior and child rearing may indirectly regulate which physical stimuli (and to what degree) impinge on the newborn and the developing infant. In this view, the brain is seen as a set of potential capacities which require and make constructive use of different patterns of physical stimulations. Cultural conventions are judged to specify when, how and to what extent an individual is to come into relation with the range of stimuli contained in the physical environment. Here, one can include physical stimuli of all types: visual (e.g. light intensity, color and form of spatial assemblages, etc.) auditory, olfactory, nociceptive, gustatory, temperature, humidity, kinesthetic, etc. Cultural conventions will also dictate diet and amount of food ingested as well as when people should eat. These types of physical environmental agents are precisely those which are manipulated by neural scientists who study environmental effects on neural development and function in vertebrate and invertebrate organisms. Moreover, to varying degrees, each (given sufficient under or overload conditions) can be expected to affect neural development and function and, by implication, behavior. The extent to which such stimuli have to be controlled in order to produce discernible effects on adult animal behavior is 
variable. More importantly whether human infants are ordinarily subject to analogous levels of "under" or "over" stimulation is questionable. Nutritional deficiencies have thus far received the greatest attention and their behavioral effects are protean. The possibility that culture, which after all is viewed by many as an adaptive system could adversely (i.e. "maladaptively") program the physical stimuli which are needed in the development of brain function raises obvious philosophical questions. None the less on logical grounds alone one should allow for this possibility. The result of such influences would be group differences in brain organization and function. Only clearly focused longitudinal studies of an interdisciplinary nature can clarify whether (and if so the extent to which) this type of cultural influence might operate.

In addition to this more or less "mechanical" way in which culture could influence adult brain organization and function, one may propose a more "dynamic" one: cultural prescriptions mediated through language are inherent in child rearing practices. Parental influences rendered in language (linguistic utterances communicating attention, reinforcement, values, etc.) reflect a code which is internalized by the infant and then helps regulate which level and/or pattern of stimuli are attended to, selected, processed and integrated in the higher levels of the nervous system. This influence might take place not in primary sensory areas but rather in secondary association areas wherein intermodal integrations and transformations are believed to take place. Initially, non-verbally (i.e. "emotively", through cooing, touch, fondling, directing, etc.) and later through actual language and speech, stimuli from the environment may be viewed as "packaged" or ordered in a meaningful way for the infant. In other words, some patterns of stimuli may be given priority and others under-emphasized; the infant learning what is important and how. In this instance, the configuration of stimuli stressed "by culture" are integral to the meanings which are accorded to them. Because of this supposition, this type of "cultural" influence is separated analytically from the preceding one. Clearly, a great deal of brain maturation takes place under the influence of social stimulations the cultural meanings of which are not yet evident to the infant. Moreover, there are obvious limits to the extent to which neural representations of social symbols can "filter" physical stimuli and "tune" the nervous system. This effect of culture on brain functioning is clearly evident in the language which the infant learns and in the socio-linguistic context of child rearing. Moreover, culture and language, together influence the music which a person comes to recognize and like, the emotions that he recognizes and the ways he shows them, conceptions of self and others, and, to some extent, the way he orients spatially. It may be that, in an analogous way, language and cultural conventions may regulate the way other brain capacities come to be realized such as those for pain behavior: in other words, neurological substrates which mediate the learning of parent-child nonverbal communications and later, actual grammatical and semantic distinctions of the natural language of the infant together with correlated behavioral influences can be viewed as affecting the way sensory information is processed. Clearly so-called 
"higher" cortical capacities (such as those underlying language) govern not only abstract (semantic) meanings, but also hedonistic values (i.e. affective, emotional) which implicate subcortical structures. In contemporary interpretations of human brain organizations emphasis is given to the various "brains" (e.g. proto-mammalian, limbic, etc.) which are seen as somehow fused together. In these accounts, emphasis is given to the role which language plays in modifying "ancestral" functions (see below) (MacLean, 1973; Isaacson, 1974).

These two views of language and cultural influences are clearly complementary. They are both held to begin early in development. They both attempt to describe how such influences might potentially affect brain maturation and neuropsychological programming in a general way. This might be accomplished, physico-chemically, through the systematic initiation of new synaptic connections or the atrophy of others (genetically determined) which somehow are played down given the prevailing adaptive requirements facing the group, or merely by repeated use of special cell assemblies and disuse of others. Finally, in potentially affecting the brain as a whole, these influences would have widespread effects in many spheres of behavior: In other words, insofar as these influences are pervasive and integral to the development of individuality they can be expected to affect brain maturation and function in a pervasive manner. It is thus not surprising that the neural representations of culture are not "localized" but wholly distributed in the brain; specifically, that patients with brain lesions in different areas still reflect the effects of culture (Fabrega, 1977; 1981).

A final way in which cultural influences might affect the neurobiology of pain is suggested by considering an adult who migrates to a new social group and comes to adopt its social practices. Such an individual will no doubt learn a great deal (non-verbally) simply by observation and imitation though his own natural language system would obviously play an influential role in this process (e.g. through verbal introspection, rehearsal and training). A better understanding of the conventions of the group would follow from learning its language. In both instances, then, neuro-linguistic substrates play a critical mediating role. Second languages seem to be represented in the same cortical centers as the original one, although there are interesting exceptions to this tendency which are not at present wellunderstood (Paradis, 1976). How and to what extent other structures and levels of the nervous system participate in this type of "enculturation" is not known. It is reasonable to assume that completely bilingual individuals show different profiles of social, cognitive and motor behaviors when they participate in different language communities. If so, impairment of one language may be associated with deficits in many areas of behavior but only when that language is called for. The literature of aphasia in bilinguals points to patterns of recovery of linguistic function but the lack of control inherent in these studies does not allow one to draw clear-cut generalizations (Paradis, 1976). Whether lesions which impair one language also influence other behaviors "natural" to that community (e.g. gesture, displays, pain 
behavior, etc.) cannot be inferred. Similarly, pain behavior in split brain subjects would offer clues about the controlling influences of language in pain.

\section{Language, Gulture and Pain: Phylogenetic Considerations}

The assumption that language and culture play an important role in brain maturation and function and by extension, in pain behavior, implies that a pure "physiology of pain" is to some extent an artifact: an abstracted "part" of a larger phenomenon which is rooted in the symbols in terms of which a people govern their way of life. Full appreciation of a phenomenon such as pain can only be gained in the light of what pain means, and how a people orient to and deal with it, all of which influence their responses. Without minimizing the validity of what is known about the neurobiology of pain, a language-culture perspective draws attention to the biocultural unity of man. A phenomenon such as pain is viewed as determined by neurobiologic substrates but elaborated by the symbol system of a people: these symbols serve to make the phenomenon cultural and in doing so necessarily shape behavior around it.

In drawing emphasis to the importance of language and culture in pain one is necessarily faced with a dilemma. The work of zoologists, experimental psychologists and ethologists abounds with the evidence that nociceptive stimulation has aversive consequences. It is even possible to apply the scientific concepts of pain threshold and tolerance to non-humans (Manning and Vierck, 1973). On intuitive grounds, one concludes that non-humans "experience" pain. It has been suggested that pain responses in higher mammals are qualitatively different from those of man. For example, under natural conditions chimpanzees appear to be less sensitive to pain than humans; but that pain responses are common occurrences is not in doubt (Mason, 1976). Yet, language and culture have been discussed here as features of human groups. This is not to deny that nonhuman primates, especially the great apes, show behaviors easily thought of as communicative, cognitive, representational and syntactical. In short, to give emphasis to linguistic and cultural aspects of pain is but to raise the question of the distinguishing properties of the human mode of adaptation, a question which cannot be discussed fully here. Important issues are said to be the human capacity to construe himself as projecting forward in time, and to be aware of his finite nature. These capacities take on added significance when one considers that humans are able to use the phenomenon of pain symbolically. Not only do pain experiences represent something (culturally) distinctive about the state of the self and the world, pain may also be handled purely as a symbolic entity. Language and culture provide for the conditions which allow man to use the concept of pain as a way of thinking about the self and world. Through metaphor, the concept of pain can be likened to other concepts about the self and the world and vice versa. The fact that in humans pain has a symbolic dimension means that it can be used 
by people as a means of creating an image about themselves. All of these aspects of pain behavior seem to exceed, in degrees of intention, selfreflection and mnemonic capacity, what higher primates seem able to achieve (given present understandings of this problem). The distinction which is made by psychologists and physiologists between sensory-discriminative as opposed to the motivational components of pain (in particular, pain as suffering) is relevant here as is the set of questions surrounding socalled psychogenic and/or hysterical pain.

Claiming that language and culture play a role in human pain can be viewed in two ways. This claim can mean that the neocortex merely registers happenings in subcortical regions which have until the advent of homo sapiens been the areas principally involved in the regulation of pain. Alternatively, the organization and regulation of pain is different structurally than it is in nonhuman primates, this difference being a consequence of newly developed neocortical functions. In either case, it is clear that an analysis of the role of language and culture in pain touches on the principle of encephalization of function (Passingham and Ettlinger, 1974; Weiskrantz, 1961; Jerison, 1976). A now outmoded interpretation of this principle states that during evolution the neural control of behavior has progressively moved in a rostral direction to the "higher" levels of the nervous system. As an example, lesions in the cortex of members of species hierarchically graded in the phylogenetic scale have seemed to produce increasingly more tangible and compromising deficits. A classical observation has been that occipital cortex lesions allegedly produced greater visual deficits in man than nonhuman primates. This led to the claim that vision was more "encephalized" in humans. Observations such as these generated the principle that during evolution the neural control of behavior had literally been shifted to the cortex. In claiming that human language and culture are influential in pain one may appear to be implying such a principle; specifically, that the neural control of pain, which in animals is located at lower levels of the nervous system, has been "transferred up" the neuraxis to the cortex in man.

However, the principle of encephalization, as stated above, has been strongly criticized and no longer seems to claim endorsement by neurobiologists. It has been rightly claimed that since there exist many graded levels of species each exhibiting many forms of behavior, such a principle as stated above would mean that many "transfers" of neural control have occurred independently. Such an interpretation of the principle of encephalization runs counter to the fundamental assumption that evolution is conservative: descendant species are far more likely to refine or build upon systems of neural control rather than to construct entirely new ones. For this reason alone, the literal shifting of neural control in species related through evolution is unlikely. An interpretation which now seems more feasible is that behavioral functions (conceivably, pain) have during evolution come to be organized differently as new ecological niches provided adaptive challenges. What one observes across grades of species is not so much "the same" brain function now regulated by newly encephalized substrates but rather 
entirely new versions of them. Such functions, though called the same (i.e. regulation of pain), now draw on forebrain structures. With regard to the regulation of pain, the claim would be that this function in humans is of a different character by virtue of evolutionary developments. Pain plays a different role (and has an elaborated structural organization in the brain) by virtue of the special mode of human adaptation which is linked to the neocortical functions of language and culture.

At issue in the controversy over the interpretation of this principle seems to be what one can term an evolutionary version of the philosophical problem of universals. Consider the following question: how useful is the claim that a particular form of behavior in different species is "the same"? (e.g. examples of "one" neural capacity or function). If such a putative capacity is seen as "the same", then given the exigencies of evolutionary theory one seems compelled to emphasize how control of that capacity "shifts" rostrally. An emphasis on the importance of language and culture would then mean that one had to explain how "older centers" of pain and pain-inhibition are now controlled by newer ones (e.g. "how the human language and association areas control phylogenetically older brain stem centers"). However, if one sees forms of behavior and neural control systems such as pain as merely similar because they draw on similar forms of physical energy, or have roughly similar consequences, then one is free to view them (pain behaviors and pain neural systems) as different solutions to basic problems of adaptation. In this latter view, evolutionary advances are associated with different realizations of a similar class of behavior and function. This would mean that culture-language (human evolutionary advances) become integral to any formulation of (human) pain. A position which ascribes to language and culture (speaking neurologically, to neocortical influences) an important role in the regulation of pain would contain the proposition that these human capacities "enter into" the phenomenon of pain and in doing so lay the groundwork for new ways of handling pain in the species.

Problems similar to those covered by the principle of encephalization (but not labeled as such) have concerned neurophysiologists and physiological psychologists. As an example, the study of the neural organization of behaviors such as pain in different species has led to the heuristic notion that man possesses three separable but complementary types of "brains". According to MacLean's triune concept, there exists a proto-reptilian brain (governing basic survival functions through more or less stereotyped behaviors based on phylogenetically old memories), a paleo-mammalian or limbic brain (which allows for the use of acquired memories of pain or pleasure) and a neo-mammalian brain (providing a refined apparatus for perceiving and interpreting the internal and external environment (MacLean, 1973). Recently, Issacson has modified and extended these notions. He judges that the proto-reptilian brain reacts to environmental changes by quantitative shifts in the intensity of responses. The paleo-mammalian or limbic brain is able to suppress protoreptilian responses thereby allowing more qualitative changes in behavior (Isaacson, 1974). 
The result of all this is that a hypothetical organism is said to be able to "forget" its old ancestral memories, thereby setting the conditions for the creation of new, acquired and more or less temporary neural associations in learning. Such learning is held to be importantly governed by the neomammalian (neocortical) brain which is associated with rapid controls, fine grained movements and precise somatotopic localization vis à vis environmental stimulation. In learning, neocortical structures speed up the excitability and conduction time of sensory systems and the formation of associations, thereby appearing to make possible whether the organism will respond on the basis of new or old information. Issacson views the control of the neocortex as providing man with a means of liberation from domination by the environment. This is illustrated in him by the unique controls provided by language and symbols. Extrapolating from this, one is able to imagine that neuro-linguistic and mnemonic functions, which take place in and/or are regulated by the cortex, are concerned with the encoding of man's symbolic systems (his language and culture). In effect this allows the meanings of these symbols to be coded neurologically and to then govern how and when limbic controls should operate. Therefore, language and culture become integral to the regulation and function of pain in man. Activities in the "older" centers of pain become modified through the special controls provided by language and culture.

\section{An Anthropological View of Psychosomatics}

The problem of how to construe or explain pain in non-humans is similar to that of providing an account of the phylogenetic roots of human pain. Just as it is difficult to specify what pain "means" to nonhuman primates, it is difficult to specify what pain meant to a pre-cultural and/or pre-linguistic hominoid. One cannot, as it were, fully understand what pain means to someone in the absence of some form of communication with that person. One can articulate neurophysiologic substrates of pain behaviors but without a common language cannot learn about its symbolic aspects. How is one to construe the gulf that separates human from nonhuman pain or make sense of the pain of pre-linguistic and pre-cultural man? More generally, how are problems of psychosomatic mediation to be construed?

One can view the human organism as a complex communication system capable of intelligence. It possesses a physical apparatus which is a product of evolution. Processes and activities which take place within and among body parts constitute a functional body system, and the human precursors of this system will be termed phylogenetic. In this "phylogenetic body system" there occur "communications" of fundamental importance to the adaptation of the species. This is to say that its body parts are connected, anatomically and functionally, in ways that promote adaptation. A human organism may also be viewed as embodying a phylogenetic psychic system whose parts are "mental" structures subserving among other things, language, perception and cognition. Again, important "communications" are 
held to take place in this phylogenetic psychic system. One may posit that a correspondence exists between these two systems and that a code of sorts provides for transformations from one to the other. Elemental features of psychosomatic mediation are thus a product of this code. In this metaphorical sense, one can say that the phylogenetic body and mind can "exchange" information and thereby reflect happenings in the other's system. This view is based on the assumption that there exists a basic set of psycho-physiological patterns which are a product of evolution and hence common to homo sapiens. An ultimate aim of inquiries in anthropology and psychosomatics is to decipher and better understand how the two phylogenetic systems function and also what the basic units of meaning of the code are which allow for psychosomatic mediation.

Every individual during the process of enculturation learns a natural language. This provides him with a means of learning what in effect is a culturally distinctive representation of the world. This world includes the physical habitat and a subset of its attributes which are selectively marked against a background which is selectively ignored; the social structures of his culture and society, which are by definition unique and distinctive; the psychological configuration (e.g. meaning, value, etc.) of the personal objects to which he relates; a set of rules, premises and accumulated truths about the world, people and how one should relate to these; and important for the psychosomaticist, a model or view of self which takes into account however crudely that which we term "body" and "mind". In brief, an individual's cultural model of self (or personhood) includes a theory about his "body" and "mind", and this model influences how he construes himself.

In the learning of language and culture a person modifies and shapes his phylogenetic psychic system. How attention comes to be deployed and how perceptual and cognitive processes come to be used are influenced by the individual's culture and natural language. The final form of an organism's psychic system is thus an organized functional system having phylogenetic and learned (i.e. linguistic-cultural) components. Besides influencing various psychic "structures" (and communications between them) culture and language are held to also influence the rest of the apparatus of man. As already stated, cultures provide explanations of "body" and through language an individual learns these. Moreover, since the individual's neuromuscular and visceral programmes mature in a learning setting molded by culture and language, the latter should also be judged to have some influence on what was termed the individual's phylogenetic "body" system. In other words, the individual learns to attend to his basic needs in a distinctive manner, and he learns an equally distinctive interpretation of his bodily states and biologic functions, and, such learning experiences take place under influences which also shape his daily activities and rhythms. The effects of language and culture, in this extended sense, encompass the body system as a whole. This is made possible, by the exchange of information which takes place through the code which is shared by the phylogenetic systems. This code itself comes to be shaped, modified and contextualized by culture, language and by the experiences which these make possible and 
indeed "embody". In this way, culture and language come to partially influence how the body functions and how mind-related happenings affect body-related happenings, and vice versa. From the standpoint of what the individual believes and feels, an important factor in the cultural patterning of mind, body and mind-body relations is the prevailing theory of self (i.e. of mind, body, personality, etc.) which the individual learns. In a sense, this cultural theory of self must come to mediate and rationalize for the individual how his mind and body interrelate. There is a dilemma in all of this. Since man is a cultural linguistic creature, the experiences of group living necessarily shapes the phylogenetic systems in a distinct way rendering them inaccessible to study. In short, though evolution furnishes us with these advanced systems, and evolution has capitalized on earlier forms of culture and language, the conditioning and contextualizing effect of natural languages and cultures is to remove the phylogenetic systems as empirical objects of study.

One can view an individual's natural language as a device for communicating with his fellows about, among other things, that to which we refer by the terms "mind" and "body". The portions of natural languages which address psychological and biological experience allow persons to talk about their likes, reasons, opinions, feelings, etc. (i.e. about their "minds") and about their internal processes, functions, dysfunctions, pains, etc. (i.e. about their "bodies"). When it comes to discourse about how the individual's apparatus functions, then, natural languages are meta-languages - that is, they are languages which allow discourse about the phylogenetic systems of "body" and "mind". In this light, the language of biomedicine (that is, the set of terms and rules of modern scientific biology which serve to explain human functioning and disease) constitutes a powerful meta-language about the body, about the mind and about mind-body relations. Furthermore, since the biomedical language allows one to talk about, compare and indeed to interpret the significance of other people's languages about their body and mind (in reality, other people's meta-language about the phylogenetic systems of the mind and body), it can function as a meta-metalanguage. There exist at least three levels of "psychosomatic discourse": the phylogenetic system of mind and body, a group's own natural (meta) language about related phenomena, and our own biomedical (meta-meta) language which allows us to analyze and compare how different people explain and talk about the mind, body and related happenings.

In an attempt to achieve some understanding of the way the "phylogenetic systems" work and of how these systems are influenced by language and culture the researcher has at his disposal a powerful descriptive and analytic device, namely that which has been termed the meta-metalanguage of biomedicine. Biomedicine allows the researcher to probe and examine the way in which mind-body relations are structured across people of different cultures and language communities. There is a dilemma here as well: just like natural languages shape the phylogenetic systems, rendering it impossible to study them in a pristine form, the biomedical meta-metalanguage, insofar as it constitutes a language, yields but a representation of 
phenomena, thereby necessarily imposing a structure on it. That is, it also selects, orders, and attributes significance to certain classes of phenomena; and, conversely, selectively relatively neglects others. The selectivity of the "biomedical meta-meta-language" has recently been emphasized dramatically as one learns about new capacities inherent in mind, body and mindbody relations (Brown, 1974). The phenomena of autonomic conditioning and acupuncture bear witness to regularities and potentialities heretofore selectively excluded by the biomedical meta-meta-language. Accurate and refined translations between and across language are, of course possible, as recent work involving biofeedback relations and current biomedical views of acupuncture amply demonstrate (e.g. involving the midbrain pain inhibition system, placebo effects, etc.) (Melzack and Jeans, 1974; Miller, 1969). However, on logical grounds, one must be prepared to accept the notion that for a range of sub-problems that fall within the concern of the researcher in comparative psychosomatics, a certain measure of incommensurability exists between languages thereby sealing off answers to certain questions one may entertain.

In claiming that the natural language and culture of an individual play a critical role in the phenomenon of pain we implicitly reflect a contemporary view to the effect that languages which are placed in widely separated linguistic groupings differ in important respects among each other, and that these differences might reflect and influence cognitive-perceptual systems and brain-behavior relations generally. Linguists of the transformationalist school acknowledge the existence of structural differences across language families; but, they view these as relatively unimportant. It is the "deeper" structure or base form of grammar which is important, and this is held to be uniform. This latter view, then, gives emphasis to universal grammatical features which underlie "surface" differences. Implied in this view is that such universals might bear a relation to the way any social and cognitive functions influenced by language might be carried out. In this transformationalist view of language it is not clear whether, and if so how, distinctive "surface" properties might influence cognition and behavior. This paper acknowledges the importance of "universals" but also views culture-specific surface properties as influential in pain behavior.

\section{References}

Beecher, H. K. (1959) "Measurement of Subjective Responses: Quantitative Effect of Drugs", Oxford University Press, New York.

Berlin, B. and Kay, P. (1969) "Basic Color Terms: Their Universality and Evolution", University of California Press, Berkeley.

Bornstein, M. H. (1973) Color vision and color naming: a psychophysiological hypothesis of cultural difference. Psychological Bulletin, 80, 257-285.

Bornstein, M. H. (1975) The influence of visual perception. American Anthropologist, 77, 774798.

Brindley, G. S. (1960) "Physiology of the Retina and the Visual Pathway", Edward Arnold, London.

Brown, B. B. (1974) "New Mind, New Body", Harper and Row, New York.

Cole, M. and S. Scribner (1974) "Culture and Thought", John Wiley and Sons, New York. 
Fabrega, H., Jr. (1974) "Disease and Social Behavior: An Interdisciplinary Perspective". MIT Press, Massachusetts.

Fabrega, H., Jr. (1975) The need for an ethnomedical science. Science, 189, 969-975.

Fabrega, H., Jr. (1977) Culture, behavior and the nervous system. Annual Review of Anthropology, 6, 419-455.

Fabrega, H., Jr. (1981) Cultural programming of brain behavior relations. In "Brain Behavior Relations” (Ed. J. Merikangas, Lexington Books, Lexington, Mass., pp. 1-64.

Fabrega, H. and Manning, P. K. (1973) An integrated theory of disease: ladino-mestizo views of disease in the Chiapas Highlands. Psychosomatic Medicine, 35, 223-239.

Fabrega, H. and Tyma, S. (1976a) Language and cultural influences in the description of pain. British Fournal of Medical Psychology, 49, 349-371, 1976.

Fabrega, H. and Tyma, S. (1976b) Culture, language and the shaping of illness: an illustration based on pain. Fournal of Psychosomatic Research, 20(4), 323--337.

Fishman, J. A. (1960) A systemization of the Whorfian hypothesis. Behavioral Science, 5, 323339.

Franklin, K. J. (1963) Kewa ethnolinguistic concepts of body parts. Southwestern Journal of Anthropology, 19, 54-63.

Heider, E. R. (1972) Universals in color naming and memory. Fournal of Experimental Psychology, 93, 10-20.

Hilgard, E. R. A neodissociation interpretation of pain reduction in hypnosis. Psychological Review, 80, 396-411.

Isaacson, R. L. (1974) "The Limbic System". Plenum Press, New York.

Jerison, H. J. (1976) Principles of the Evolution of the Brain and Behavior. In "Evolution, Brain and Behavior: Persistent Problems" (Eds R. B. Masterton, W. Hodos, and H. Jerison) Lawrence Erlbaum Associates, New Jersey, pp 23-45.

Lewis, B. (1975) "Knowledge of Illness in a Sepik Society: A Study of the Gnau, New Guinea". The Athlone Press, London.

Liebeskind, J. C. and Paul, L. A. (1977) Psychological and physiological mechanisms of pain. Annual Review of Psychology, 28, 41-60.

MacLean, P. D. (1973) "A Triune Concept of the Brain and Behavior" (Eds T. J. Boag and D. Campbell). University of Toronto Press, Canada.

Mandler, G. (1967) The conditions for emotional behavior. In "Biology and Behavior: Neurophysiology and Emotion" (Ed. D. C. Glass) Rockefeller University Press, New York, pp. 96-102.

Manning, A. A. and Vierck, C. J., Jr. (1973) Behavioral assessment of pain detection and tolerance in monkeys. Fournal of the Experimental Analysis of Behavior, 19(1), 125-132.

Mason, W. A. (1976) Environmental models and mental modes: representational processes in the great apes and man. American Psychologist, 31, 284-294.

Mayer, D. J. and Price, D. D. (1976) Central nervous system mechanisms of analgesia. Pain, 2, 379-404.

Melzack, R. and Jeans, M. (1974). Acupuncture analgesia: a psychophysiological explanation. Minnesota Medicine, 57, 161-166.

Miller, N. E. (1969) Learning of visceral and glandular responses. Science, 163, 434-445.

Nathan, P. W. (1976) The gate-control theory of pain. Brain, 99, 123-158.

Paradis, M. (1976) Bilingualism and aphasia. In "Studies in Neurolinguistics" (Eds. H. Whitaker and H. Whitaker). Academic Press, New York.

Passingham, R. E. and Ettlinger, G. (1974) A comparison of cortical functions in man and other primates. International Review of Neurobiology, Vol. 16.

Pike, K. L. (1967) "Language in Relation to a Unified Theory of the Structure of Human Behavior". Mouton and Co., The Hague.

Pike, K. L. (1975) "On Describing Language”, The Peter DeRidder Press, Lisse.

Pike, K. L. and Pike, E. (1977) "Grammatical Analysis", Summer Institute of Linguistics, California.

Ruesch, J. and Bateson, G. (1968) "Communication: The Social Matrix of Psychiatry". W. W. Norton and Co., New York.

Sahlins, M. (1976) Colors and Cultures. Semiotica, 16, 1-22. 
Sapir, E. (1927) The unconscious patterning of behavior in society. In "Unconscious: A Symposium" (Ed. E. S. Dummer) Knopf, New York pp. 114-142.

Warrington, E. G. (1975) The selective impairment of semantic memory. Quarterly Journal of Experimental Psychology, 27, 635-657.

Weiskrantz, L. (1961) Encephalization and the scotoma. In "Current Problems in Animal Behavior" (Eds W. H. Thorpe and O. L. Zangwill). Cambridge University Press, London, pp. 30-58.

Whorf, B. L. (1964) "Language, Thought and Reality", MIT Press, Massachusetts.

Wolff, B. B. and Langley, S. (1968) Gultural factors and the response to pain: a review. American Anthropologist, 70, 494-501.

Zborowski, M (1958) Cultural components in responses to pain. In "Patients, Physicians and Illness" (Ed. E. G. Jaco). Free Press of Glencoe, New York, pp. 256-268.

Zola, I. K. (1966) Culture and symptoms: an analysis of patients' presenting complaints. American Sociological Review, 31, 615-630. 


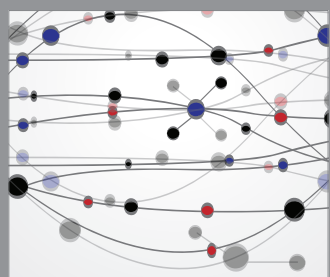

The Scientific World Journal
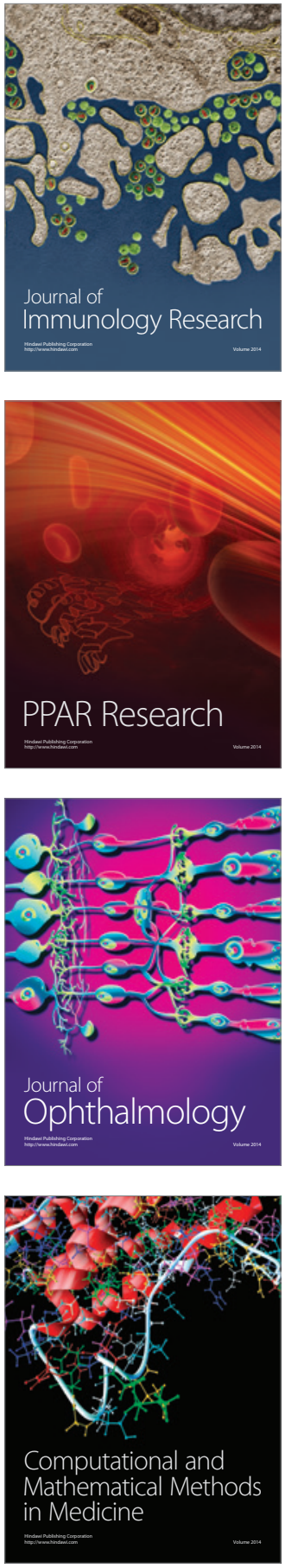

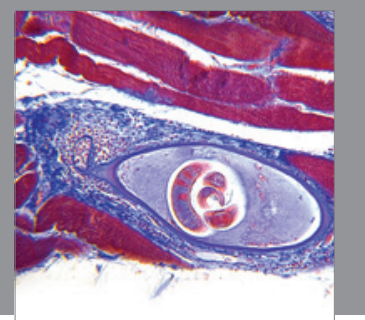

Gastroenterology

Research and Practice
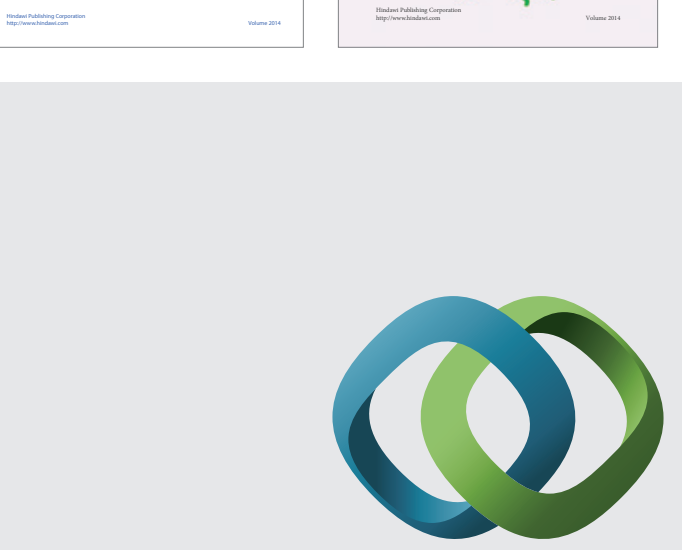

\section{Hindawi}

Submit your manuscripts at

http://www.hindawi.com
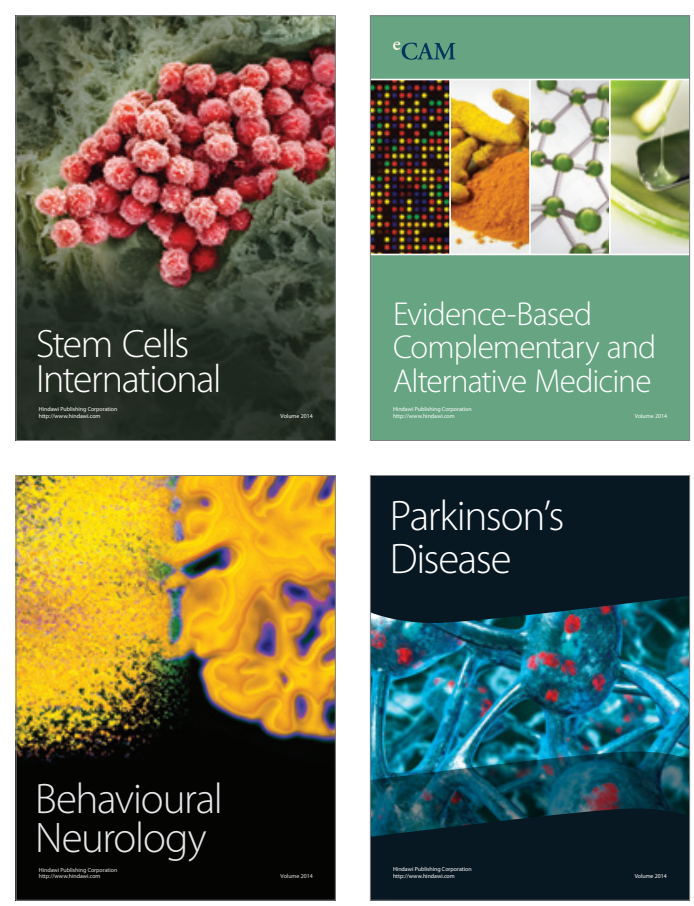

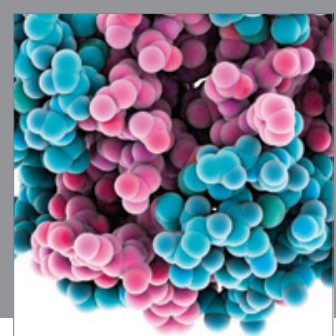

Journal of
Diabetes Research

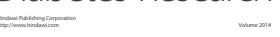

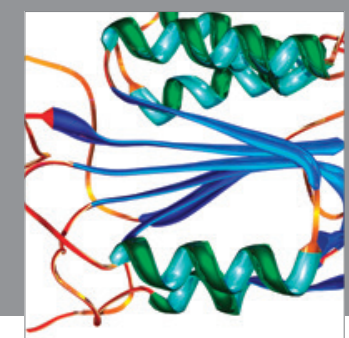

Disease Markers
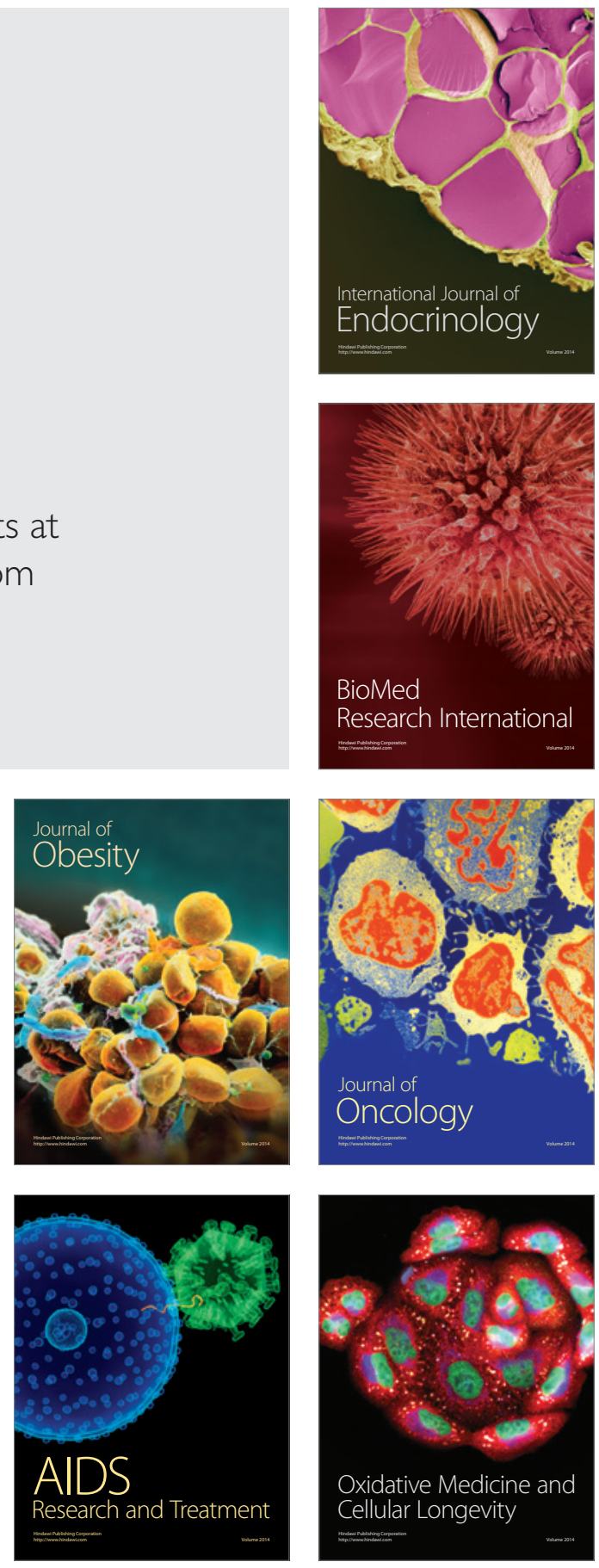